International Mathematical Forum, 2, 2007, no. 26, 1295 - 1303

\title{
On Generalized Interval Orders
}

\author{
Athanasios Andrikopoulos ${ }^{1}$ and Eleni Philippopoulou
}

\author{
Department of Economics \\ University of Ioannina \\ 45110 Ioannina, Greece
}

\begin{abstract}
In this paper, we introduce the notion of generalized interval order (GIO) which extends the notion of interval order in non-transitive binary relations. This allow us to extend the classical representation theorem of Fishburn in [5]. We also provide sufficient conditions which ensure the existence of the Generalized Optimal Choice Set (GOCS) of GIOs. Finally, we characterize the existence of the GOCS of GIOs.
\end{abstract}

Keywords: Interval order, Maximal elements

\section{Introduction}

A partially ordered set $(X, \succ)$ is called an interval order if there is a function $I$ assigning to each element $x \in X$ a closed interval $I(x)=\left[m_{x}, M_{x}\right]$ of the real line $\mathbb{R}$ so that for all $x, y \in X, y \succ x$ if and only if $m_{y}>M_{x}$ in $\mathbb{R}$. The concept of an interval order was introduced by Peter C. Fishburn in [5] to build models of preferences whose associated indifferences may fail to be transitive. Comparing intervals is a frequently encountered problem in preference modelling, decision aid and experimental psychology. This is due to the fact that the comparison judgments in experimental psychology (perception, evaluation of objects, persons) as well as the comparison of alternatives in economic theory (outcomes, objects, candidates, etc) generally are realized through their evaluations on numerical scales, while such evaluations often are imprecise or uncertain. Thus, taking an example from economic theory, an interval order is obtained when one considers that an alternative is preferred to another iff its interval is "completely to the right" of the other, while any two alternatives, the interval of which have a non-empty intersection are considered indifferent. A classical result in interval orders is that of Fishburn in [5]; which examines the issue of representing an interval order $\succ$ by means of two functions $u$ and $v(x \succ y$ if and only if $u(x)>v(y))$. Numerical representations are used in

\footnotetext{
12aandriko@cc.uoi.gr
} 
order to look for maximal elements of binary relations in the set of alternatives. Mathematical optimization tools are applied more easily with numerical representations of binary relations. Existence of maximal elements of binary relations has interested economists for long. This kind of relations appear e.g. in preference theory, in equilibrium theory and in the analysis of dynamic systems. In all of these choice problems, the interpretation of existence of maximal elements means that there exists a choice for which there exist no strictly better choices. In the case of considering the set of alternatives as a topological space, conditions for the existence of maximal elements are given by using topological conditions on the alternative's set as well as continuity assumptions on the relation. One of the more general continuity assumption on finding the maximal choice set of an acyclic binary relation $R$, is that of upper tc-semicontinuity defined by Alcantud and Rodríguez-Palmero in [1]. This definition uses the transitive closure of the relation $R$. As the set of maximal choices (maximal choice set) is empty and, hence, does not help much when $R$ contains a top cycle ${ }^{2}$, we turns to the concept of Generalized Optimal Choice Set (GOCS), which is a generalization of the maximal choice set. The origin of GOCS can be found in Schwartz [7]. Schwartz proved that the GOCS is the maximal choice set of the asymmetric part of the transitive closure of $R$ (cf. [14, Corollary 6.2.2]). Van Deemen in [9] proves that if the set of alternatives is finite, then the GOCS is never empty. The existence of maximal elements for interval orders have been mainly investigated by Campbell and Walker in [4] and Alcantud in [2]. More precisely, Campbell and Walker provide sufficient conditions to ensure the existence of maximal elements for interval orders and Alcantud characterize the existence of maximal elements of interval orders.

In this paper, we extend the notion of interval order in non-transitive binary relations. This new notion which is called generalized interval order it allows us to examine the representation of binary relations by means of two functions, in a general framework, where the axioms of transitivity and irreflexivity do not hold. Here, we provide a generalization of the classical result of Fishburn in [5]. Finally, we use the notion of GOCS set (which generalize the notion of "maximal choice set"), as it is defined by Schwartz in [7] and we extend the results of Campbell and Walker in [4] and Alcantud in [2]. All of the results known in the literature about representations of binary relations and the existence of their maximal elements, make use of conditions (e.g. continuity conditions) based either on the binary relation itself or on its transitive closure. After the above mentioned result of Schwartz, a natural generalization of the

\footnotetext{
${ }^{2}$ Consider a commodity space $X$ and a binary relation $R$ defined on $X$. A path from $x$ to $y$ is a finite sequence of distinct points $x_{1}, x_{2}, \ldots, x_{n}$ such that $x R x_{1}, x_{1} R x_{2}, \ldots, x_{n} R y$. A path from $x$ to $y$ becomes a cycle when the pair $y R x$ is added. The finite sequence $\left(x_{1}, x_{2}, \ldots, x_{n}\right)$ is a top cycle in $X$ iff (i) $\left(x_{1}, x_{2}, \ldots, x_{n}\right)$ is a cycle in $X$ and (ii) there is no $x \in X \backslash\left\{x_{1}, x_{2}, \ldots, x_{n}\right\}$ and there is no $y \in\left\{x_{1}, x_{2}, \ldots, x_{n}\right\}$ such that $x R y$.
} 
well known notions (e.g. weakly upper semicontinuity), which will ensure the compatibility of the extended results with the classical ones, is to replace the transitive closure of a binary relation with the asymmetric part of the transitive closure of this relation. This generalization transform the problem of the existence of GOCS into the problem of the existence of maxima of a real-valued function.

\section{Notations and Definitions}

We recall some definitions from [1], [5], [8].

Let $X$ be a non-empty universal set of alternatives, and let $R \subseteq X \times X$ be a binary relation on $X$. The asymmetric part $P(R)$ of $R$ is denoted by $P(R)=$ $\{(x, y) \in X \times X:(x, y) \in R$ and $(y, x) \notin R\}$. We sometimes abbreviate $(x, y) \in R$ as $x R y$. The binary relation $R$ is a strict partial order if it is irreflexive (we never have $(x, x) \in R$ ) and transitive (for all $x, y, z \in X, x R z R y$ implies $x R y)$. We often use $\succ$ to denote a strict partial order.

The transitive closure of a relation $R$ is denoted by $\bar{R}$, that is for all $x, y \in$ $X,(x, y) \in \bar{R}$ if there exists $k \in \mathbb{N}$ and $x_{0}, \ldots, x_{K} \in X$ such that $x=x_{0}$ $\left(x_{k-1}, x_{k}\right) \in R$ for all $k \in\{1, \ldots, K\}$ and $x_{K}=y$. Clearly, $\bar{R}$ is transitive and, because the case $K=1$ is included, it follows that $R \subseteq \bar{R}$. Acyclicity says that there do not exist $K$ and $x_{0}, x_{1}, \ldots, x_{K}$ such that $x=x_{0},\left(x_{k-1}, x_{k}\right) \in R$ for all $k \in\{1, \ldots, K\}$ and $x_{K}=x$. An element $x^{*} \in X$ is said to be a maximal element of the binary relation $R$ on $X$, if $\left(y, x^{*}\right) \in P(R)$ does not hold for all $y \in X$. According to Schwartz, the Generalized Optimal Choice Set (GOCS) of a binary relation $R$ is defined to be the maximal choice set of the asymmetric part of the transitive closure of $R(P(\bar{R})$ in symbols). It is clear that for each $R \subseteq X \times X, P(\bar{R})$ defines a strict partial order on $X$. An irreflexive binary relation $R$ is an interval order if it satisfies the following interval order condition: $\left(x, x^{\prime}\right) \in R,\left(y^{\prime}, y\right) \in R$ and $\left(y^{\prime}, x^{\prime}\right) \notin R$ imply $(x, y) \in R$. Irreflexivity and the interval order condition imply that $R$ is transitive.

An asymmetric binary relation $R(P(R)=R)$ is weakly upper semicontinuous if whenever $x R y$ there is a neighborhood $U$ of $y$ such that $z R x$ is false for each $z \in U$. Associated with $R$ we consider the binary relation $\sim$ defined as $x \sim y$ if neither $x R y$ nor $y R x$. The relation $\sim$ is said to be the indifference associated to $R$. The strong indifference relation $\approx$ for $R$ is defined by $x \approx y$ if for all $z \in X, x \sim z \Leftrightarrow y \sim z$.

Definition 1 A binary relation $R$ on $X$ satisfies the transitive (tc)-interval order condition if and only if: for each $x, y, x^{\prime}, y^{\prime} \in X$ if $\left(x, x^{\prime}\right) \in \bar{R}$ and $\left(y^{\prime}, y\right) \in \bar{R}$ then $(x, y) \in \bar{R}$ or $\left(y^{\prime}, x^{\prime}\right) \in \bar{R}$. In this case, $R$ is called generalized interval order. 
An equivalent expression for the tc-interval order condition is the following:

$$
\left(x, x^{\prime}\right) \in \bar{R},\left(y^{\prime}, y\right) \in \bar{R} \text { and }\left(y^{\prime}, x^{\prime}\right) \notin \bar{R} \Rightarrow(x, y) \in \bar{R}
$$

Examples 1 (i) A non-irreflexive or non-transitive binary relation satisfing the interval order condition is a generalized interval order which is not an interval order (cf. Proposition 6 below). (ii) Let $X=\{1,2,3,4\}$ and $R=$ $\{(1,1),(1,2),(2,3),(3,4)\}$. Then $R$ is a non-irreflexive, non-transitive generalized interval order which does not satisfies the interval order condition.

Definition 2 Let $R$ be a binary relation on $X$. Then the relation $\sim_{R}$ of $R$ indifference and the relation $\approx_{R}$ of strong $R$-indifference are defined as follows:

$$
x \sim_{R} y \text { if neither } x P(\bar{R}) y \text { nor } y P(\bar{R}) x
$$

and

$$
x \approx_{R} y \text { if for all } z \in X, x \sim_{R} z \Leftrightarrow y \sim_{R} z .
$$

If $R$ is a strict partial order then the notions $R$-indifference and strong $R$-indifference coincide, respectively, with the classical notions of indifference and strong indifference.

If $\approx$ is an equivalence relation on a topological space $(X, \tau)$, then the quotient set by this equivalence relation $\approx$ will be denoted by $\frac{X}{\approx}$, and its elements (equivalence classes) by $[x]_{\approx}$. Let the projection map $\pi \stackrel{\approx}{:} X \longrightarrow \frac{X}{\approx}$ which carries each point of $X$ to the element of $\frac{X}{\approx}$ that contains it. In the quotient topology induced by $\pi$, a subset $U$ of $\frac{X}{\approx}$ is open in $\underset{\approx}{\approx}$ if and only if $\pi^{-1}(U)$ is open in $X$. Thus, the typical open set in $\underset{\approx}{\approx}$ is a collection of equivalence classes whose union is an open set in $X$. The quotient topology associated with a topological space $(X, \tau)$ and an equivalence relation $\approx$ will be denoted by $\tau_{\text {quot }}(\approx)$. The equivalent relation $\approx$ on $X$ saturates an $A \in \tau$ if $x \in A$ and $y \approx x$ implies $y \in A$. In this case we say that $A$ is a $\approx$-saturated set. If $\pi$ is the projection map associated with the equivalence relation $\approx$ on $X$, then the condition $A=\pi^{-1}(\pi(A))$ for $A \in \tau$ is equivalent to $\approx$ saturates $A$.

Definition 3 A binary relation $R$ is $\approx$-saturated weakly upper semicontinuous (SWUS), if whenever $x P(\bar{R}) y$ there is a $\approx$-saturated open neighborhood $U$ of $y$ such that $z \bar{R} x$ is false for each $z \in U$.

Finally, we define a binary relation $\widehat{R}$ on $\frac{X}{\approx_{R}}$ by :

$$
[x]_{\approx_{R}} \widehat{R}[y]_{\approx_{R}} \text { if there are } x^{\prime} \in[x]_{\approx_{R}}, y^{\prime} \in[y]_{\approx_{R}} \text { such that }\left(x^{\prime}, y^{\prime}\right) \in P(\bar{R})
$$

We call $\widehat{R}$, the $\approx_{R}$-quotient relation of $R$. 


\section{Results}

As we mention in the introduction, we are interested to extend the classical result of Fishburn in [5] to the class of generalized interval orders. First, we give three auxiliary propositions.

Proposition 2 Let $R$ be a binary relation on $X$. Then $x P(\bar{R}) z$ and $z \approx_{R} y$ imply $x P(\bar{R}) y$ and $x \approx_{R} z$ and $z P(\bar{R})$ y imply $x P(\bar{R}) y$.

Proof. We prove that $x P(\bar{R}) z$ and $z \approx_{R} y$ imply $x P(\bar{R}) y$. The rest is proved similarly. Suppose that $x P(\bar{R}) z$ and $z \approx_{R} y$. Then $(y, x) \notin P(\bar{R})$. Suppose that $(x, y) \notin P(\bar{R})$. Then $x \sim_{R} y$. Hence, from $z \approx_{R} y$ we conclude that $x \sim_{R} z$ which contradicts $x P(\bar{R}) z$. Thus, $x P(\bar{R}) y$.

Proposition 3 Let $R$ be a binary relation defined on a nonempty set $X$. If $R$ satisfies the interval order condition, then $R$ is a generalized interval order.

Proof. Let $R$ be a binary relation and let $x, x^{\prime}, y, y^{\prime} \in X$ such that $\left(x, x^{\prime}\right) \in \bar{R}$, $\left(y^{\prime}, y\right) \in \bar{R}$ and $\left(y^{\prime}, x^{\prime}\right) \notin \bar{R}$. Then, there exist $K \in \mathbb{N}$ and $x_{0}, \ldots, x_{K} \in X$ such that $x=x_{0},\left(x_{k-1}, x_{k}\right) \in R$ for all $k \in\{1, \ldots, K\}$ and $x_{K}=x^{\prime}$. There also exist $M \in \mathbb{N}$ and $y_{0}, \ldots, y_{M} \in X$ such that $y^{\prime}=y_{0},\left(y_{m-1}, y_{m}\right) \in R$ for all $m \in\{1, \ldots, M\}$ and $y_{M}=y$. Then, from $\left(x_{K-1}, x^{\prime}\right) \in R,\left(y^{\prime}, y_{1}\right) \in R$ and $\left(y^{\prime}, x^{\prime}\right) \notin R$ we conclude that $\left(x_{K-1}, y_{1}\right) \in R$. Hence, $(x, y) \in \bar{R}$.

If $R$ is a partial order, then the notions of generalized interval order and interval order coincide.

Proposition 4 If $R$ is a generalized interval order defined on a nonempty set $X$, then $P(\bar{R})$ is an interval order on $X$.

Proof. It is clear that $P(\bar{R})$ is irreflexive. Let now $x, x^{\prime}, y, y^{\prime} \in X$ such that $\left(x, x^{\prime}\right) \in P(\bar{R}),\left(y^{\prime}, y\right) \in P(\bar{R})$ and $\left(y^{\prime}, x^{\prime}\right) \notin P(\bar{R})$. We must prove that $(x, y) \in P(\bar{R})$. We have two cases:

Case 1. $\left(y^{\prime}, x^{\prime}\right) \notin \bar{R}$. Then, by $\left(x, x^{\prime}\right) \in \bar{R}$ and $\left(y^{\prime}, y\right) \in \bar{R}$ we conclude that $(x, y) \in \bar{R}$. Suppose that $(y, x) \in \bar{R}$. Then $\left(y^{\prime}, x^{\prime}\right) \in \bar{R}$ which is impossible. Hence $(x, y) \in P(\bar{R})$.

Case 2. $\left(x^{\prime}, y^{\prime}\right) \in \bar{R}$ and $\left(y^{\prime}, x^{\prime}\right) \in \bar{R}$. Then $(x, y) \in \bar{R}$. If $(y, x) \in \bar{R}$, then $\left(y, y^{\prime}\right) \in \bar{R}$ which is impossible. Hence $(x, y) \in P(\bar{R})$.

The proof of the following theorem which generalize the main result of Fishburn in [5], is inspired by the result of Bridges in [3] for interval orders.

Theorem 5 Let $R$ be a binary relation on a set $X$ such that $\frac{X}{\approx_{R}}$ is countable. Then the following conditions are equivalent:

(i) $R$ is a generalized interval order. 
(ii) There exist mappings $u, v: X \rightarrow \mathbb{R}$, with $v \geq u$, such that, for all $x, y \in X$

$$
x P(\bar{R}) y \text { if and only if } u(x)>v(y) .
$$

iii) There exist mappings $u, \rho: X \rightarrow \Re$, with $\rho>0$, such that, for all $x, y \in X$

$$
x P(\bar{R}) y \text { if and only if } u(x)>u(y)+\rho(y) .
$$

Proof. $\quad(i i i) \Rightarrow(i i) \Rightarrow(i)$ is evident. We prove that $(i) \Rightarrow($ iii $)$. Let $R$ be a generalized interval order on $X$, and let $\frac{X}{\approx_{R}}=\left\{\left[x_{0}\right]_{\approx_{R}},\left[x_{1}\right]_{\approx_{R}}, \ldots\right\}$. Let also $\widehat{R}$ be the $\approx_{R^{\text {-quotient }}}$ relation of $R$. Then, by Proposition 5 , it is easy to verify that

$$
x P(\bar{R}) y \quad \text { if and only if } \quad[x]_{\approx_{R}} \widehat{R}[y]_{\approx_{R}} .
$$

Hence, $\widehat{R}$ is a strict partial order in $\frac{X}{\approx_{R}}$. By Proposition $7, \widehat{R}$ also satisfies the interval order condition in $\frac{X}{\approx_{R}}$. For each $[x]_{\approx_{R}}$ in $\frac{X}{\approx_{R}}$, we define $S\left([x]_{\approx_{R}}\right)=\left\{n \in \mathbb{N}:\left([x]_{\approx_{R}},\left[x_{n}\right]_{\approx_{R}}\right) \in \widehat{R}\right\}$ and $T\left([x]_{\approx_{R}}\right)=\{n \in \mathbb{N}: \exists m \in$ $\mathbb{N},\left(\left[x_{m}\right]_{{\tau_{R}}_{1}},[x]_{{\approx_{R}}_{R}}\right) \notin \widehat{R}$ and $\left.\left(\left[x_{m}\right]_{{\tau_{R}}_{R}},\left[x_{n}\right]_{{\tau_{R}}_{R}}\right) \in \widehat{R}\right\}$. Define also, $\bar{u}\left([x]_{{\approx_{R}}_{R}}\right)=$ $\sum_{n \in S\left([x]_{\approx_{R}}\right)} 2^{-n}$ if $S\left([x]_{\approx_{R}}\right) \neq \varnothing$ and $\bar{u}\left([x]_{\approx_{R}}\right)=0$ if $S\left([x]_{\approx_{R}}\right)=\varnothing$ as well as $\bar{v}\left([x]_{\approx_{R}}\right)=\sum_{n \in T\left([x]_{\approx_{R}}\right)} 2^{-n}$ if $T\left([x]_{\approx_{R}}\right) \neq \varnothing$ and $\bar{v}\left([x]_{\approx_{R}}\right)=0$ if $T\left([x]_{\approx_{R}}\right)=\varnothing$. For $[x]_{\approx_{R}}=\left[x_{j}\right]_{{\approx_{R}}_{R}}$ we put $\bar{\rho}\left([x]_{{\approx_{R}}_{R}}\right)=2^{-j-1}+\bar{v}\left([x]_{\approx_{R}}\right)-\bar{u}\left([x]_{\approx_{R}}\right)$. Clearly, $S\left([x]_{\approx_{R}}\right) \subseteq T\left([x]_{\approx_{R}}\right)$; so that $\bar{v}\left([x]_{\approx_{R}}\right) \geq \bar{u}\left([x]_{\approx_{R}}\right)$, and therefore $\bar{\rho}\left([x]_{\approx_{R}}\right)>0$.

Let $x, y \in X$ such that $x P(\bar{R}) y$ and let $[y]_{{\tau_{R}}_{R}}=\left[x_{j}\right]_{\approx_{R}}$ for some $j \in \mathbb{N}$. Then, $[x]_{{\approx_{R}}_{R}} \widehat{R}\left[x_{j}\right]_{{\approx_{R}}_{R}}$, and therefore $T\left([y]_{\approx_{R}}\right) \subseteq S\left([x]_{\approx_{R}}\right)$.

From $j \in \bar{S}\left([x]_{\approx_{R}}\right) \backslash \bar{T}\left([y]_{\approx_{R}}\right)$ we conclude that

$$
\bar{u}\left([x]_{\approx_{R}}\right) \geq\left(\bar{v}[y]_{\approx_{R}}\right)+2^{-j}>\bar{u}\left([y]_{\approx_{R}}\right)+\bar{\rho}\left([x]_{\approx_{R}} .\right.
$$

Let $\pi: X \longrightarrow \frac{X}{\approx_{R}}$ be the projection map. We put $u=\bar{u} \circ \pi, v=\bar{v} \circ \pi$ and $\rho=\bar{\rho} \circ \pi$. Then,

$$
u(x)=\bar{u} \circ \pi(x)=\bar{u}\left([x]_{\approx_{R}}\right)>\bar{u}\left([y]_{\approx_{R}}\right)+\bar{\rho}\left([y]_{\approx_{R}}\right)=u(y)+\rho(y)
$$

On the other hand, if $(x, y) \notin P(\bar{R})$, then $S\left([x]_{\approx_{R}}\right) \subseteq T\left([y]_{\approx_{R}}\right)$, and so

$$
u(y)+\rho(y)=\bar{u}\left([y]_{\approx_{R}}\right)+\bar{\rho}\left([y]_{\approx_{R}}\right) \geq \bar{u}\left([x]_{\approx_{R}}\right)=u(x) .
$$

Hence, $u(x) \ngtr u(y)+\rho(y)$ which implies that

$$
x P(\bar{R}) y \Leftrightarrow u(x)>u(y)+\rho(y) .
$$

As a corollary of the above Theorem, we have the main theorems of Fishburn in [5] and Bridges in [3]. 


\section{Characterization of the existence of Gener- alized Optimal Choice Set of GIOs}

We extend the notion of $R$-maximal element defined by Suzumura in [8], as follows:

Definition 4 An element $x^{*} \in X$ is said to be an optimal element of the binary relation $R$ on $X$, if $\left(y, x^{*}\right) \in P(\bar{R})$ does not hold for all $y \in X$.

The set of optimal elements in $X$ constitutes its GOCS. In transitive binary relations the notion of optimal element coincides with the notion of $R$-maximal element. In order to characterize the existence of optimal elements in GIOs we need the following theorem of Campbell and Walker in [4].

Theorem 6 Let $(X, \tau)$ be a compact topological space. Then, every weakly upper semicontinuous interval order on $X$ has a maximal element.

Lemma 7 Let $R$ be a binary relation defined on a set $X$ and let $\widehat{R}$ be the $\approx_{R^{-}}$quotient relation of $R$ on $\frac{X}{\approx_{R}}$. A $[z]_{\approx_{R}}$ in $\frac{X}{\approx_{R}}$ is a maximal element on $\frac{X}{\approx_{R}}$ if and only if each $t \in[z]_{\approx_{R}}$ is an optimal element on $X$.

Proof. Let $\left[x^{*}\right]_{\approx_{R}}$ be a maximal element on $\frac{X}{\approx_{R}}$. If we suppose that a $t \in\left[x^{*}\right]_{\approx_{R}}$ is not an optimal element on $X$, then there is some $y \in X$ such that $y P(\bar{R}) t$. Hence $[y]_{\approx_{R}} \widehat{R}\left[x^{*}\right]_{\approx_{R}}$, a contradiction. Hence, $t$ is an optimal element of $X$.

Theorem 8 Let $(X, \tau)$ be a compact topological space, and let $R$ be a $\approx_{R^{-}}$ saturated weakly upper semicontinuous GIO. Then, the Generalized Optimal Choice Set of $R$ is non-empty.

Proof. Firstly, we shall show that $\widehat{R}$ in $\left(\frac{X}{\approx_{R}}, \tau_{\text {quot }}\left(\approx_{R}\right)\right)$ satisfies the suppositions of Theorem 11. Indeed, $\widehat{R}$ is a partial order and by definition of $\left.\tau_{\text {quot }}\left(\approx_{R}\right)\right)$, the space $\left(\frac{X}{\approx_{R}}, \tau_{\text {quot }}\left(\approx_{R}\right)\right)$ is compact. By Proposition 7 , we also conclude that $\widehat{R}$ satisfies the interval order condition. Finally, $\widehat{R}$ is weakly upper semicontinuous. To check it, let $[x]_{\approx_{R}} \widehat{R}[y]_{\approx_{R}}$ for some $[x]_{\approx_{R}},[y]_{\approx_{R}} \in \frac{X}{\approx_{R}}$. Then $x P(\bar{R}) y$. Hence, there is a $\approx_{R^{-}}$saturated open neighborhood $U$ of $y$ such that $z \bar{R} x$ is false for each $z \in U$. Hence, $\pi^{-1}(\pi(U))=U$ which implies that $\pi(U)$ is $\tau_{\text {quot }}\left(\approx_{R}\right)$ )-open neighborhood of $[y]_{\approx_{R}}$. Thus, $\approx_{R}$ satisfies the weakly upper semicontinuity condition $\left([z]_{\approx_{R}} \widehat{R}[x]_{\approx_{R}}\right.$ is false for each $\left.[z]_{\approx_{R}} \in \pi(U)\right)$. Hence, by Theorem 10 , we conclude that $\widehat{R}$ has a maximal element $\left[x^{*}\right]_{\approx_{R}}$ on 
$\left(\frac{X}{\approx_{R}}, \tau_{\text {quot }}\left(\approx_{R}\right)\right)$. Then, Lemma 11 implies that the set of optimal elements is non-empty.

We now use Theorem 12 in order to prove the following characterization of the existence of optimal elements in the class of GIOs, which extends the Theorem 7 of Alcantud in [2].

Theorem 9 Let $R$ be a GIO defined on $X$. The following conditions are equivalent:

(a) The Generalized Optimal Choice Set of $R$ is non-empty,

(b) there exists a compact topology on $X$ such that $R$ is $\approx_{R}$-saturated weakly upper semicontinuous.

Proof. (b) implies (a) by Theorem 12. We now prove that (a) implies (b).

Let $\mathcal{M}$ be the set of optimal elements (GOCS) in $X$. Let $\tau$ be the excluded set topology generated by $\mathcal{M}[6$, p.48] (this has as open sets all those subsets of $X$ which are disjoint from $\mathcal{M}$, together with $X$ itself). Then $X$ is compact under $\tau$ since every open cover of $X$ includes $X$ itself. Hence, $\{X\}$ is always a finite subcover. It remains to prove that $R$ is $\approx_{R}$-saturated weakly upper semicontinuous. Indeed, let $x, y \in X$ such that $x P(\bar{R}) y$. Then, for each $z \in \mathcal{M}$, the set $K=\{t \in X: x P(\bar{R}) t\}$ does not contain $z$. Hence, $\mathcal{M} \cap K=\emptyset$. Thus, $K$ is an open neighborhood of $y$. It is clear that for each $t \in K,(t, x) \notin$ $\bar{R}$. Indeed, if $(t, x) \in \bar{R}$ for some $t \in K$, then $t P(\bar{R}) x P(\bar{R}) t$ or $(x, t) \in I(\bar{R})$ which implies that $(x, t) \notin P(\bar{R})$, an absurdity. Finally, Proposition 5 implies that $K$ is $\approx_{R}$-saturated.

\section{References}

[1] Alcantud J., Rodriguez-Palmero,C., Orderability of topological spaces by continuous preferences, Int. J. Math. Math. Sci., 27, (2001), 505-512.

[2] Alcantud J., Characterization of the existence of maximal elements of acyclic relations, Econ. Theory., 19, (2002), 407-416.

[3] Bridges D., Numerical representation of intransitive preference on a countable set, J. Econ. Theory, 30, (1983), 213-217.

[4] Campbell, D. E., Walker, M., Maximal elements of weakly continuous relations, J. Econ. Theory, 50, (1990), 459-464.

[5] Fishburn P., Intransitive indifference with unequal indifference intervals, $J$. Math. Psychology, (1970), 144-149.

[6] Lynn, A. S., J. Arthur Seebach, Counterexamples in Topology, Campbell, D. E., Springer-Verlag, New York, (1978). 
[7] Schwartz, T., The logic of Collective Choice, New York: Columbia University Press.

[8] Suzumura K., On constrained dual recoverability theorems, Math. Soc. Sci., 45, (2002), 143-154.

[9] Van Deemen M.A., Coalition formation and social choice, Academic Publishers, Dordrecht, 1997.

Received: October 21, 2006 